\title{
Type I Bismuth-Corlette Hilar Cholangiocarcinoma
}

National Cancer Institute

\section{Source}

National Cancer Institute. Type I Bismuth-Corlette Hilar Cholangiocarcinoma. NCI

Thesaurus. Code C134737.

Tumor is limited to the common hepatic duct, below the level of the confluence of the right and left hepatic ducts. (AJCC 8th ed.) 